\title{
MÉTODO PARA COLETA DE TEMPOS E TRAJETOS EM ESCADAS PARA A OBTENÇÃO DE VELOCIDADE DE CAMINHAMENTO DE CRIANÇAS EM SIMULADOS DE ABANDONO $^{1}$
}

\author{
Rosaria Ono ${ }^{2}$ \\ Marcos Vargas Valentim ${ }^{3}$
}

\section{RESUMO}

Este artigo tem por objetivo apresentar os métodos utilizados para coletar os tempos nos pontos predefinidos (início e fim do lance das escadas) e os trajetos percorridos pelos alunos, tanto nos lances quanto nos patamares das escadas, e, consequentemente, as distancias percorridas em função do trajeto utilizado, em um simulado de abandono. Serão apresentadas as equações desenvolvidas para calcular o trajeto nos patamares. Essas equações tiveram como parâmetros, além das dimensões das escadas, a medida bideltóide média dos alunos. A pesquisa foi desenvolvida na cidade de São Paulo, Brasil, no ano de 2017. Foram utilizadas, para a coleta dos dados, as filmagens do circuito fechado de televisão (CFTV) instalado para esse fim. Participaram da pesquisa 164 alunos, com idade entre 6 e 10 anos, de uma mesma escola. A quantidade de trajetos coletados nos lances foi de 656 e, nos patamares, de 492. O movimento nos lances e patamares foram analisados separadamente e foi possível obter a frequência de trajetos percorridos e as velocidades tanto nos lances quanto nos patamares da escada.

Palavras-chave: Simulações de abandono, saídas de emergência, rotas de fuga, velocidade de caminhamento, crianças.

\footnotetext{
${ }^{1}$ Trabalho originalmente publicado nos Anais do 5 CILASCI - Congresso Ibero-LatinoAmericano sobre Segurança contra Incêndio.

${ }^{2}$ Professora Titular na Faculdade de Arquitetura e Urbanismo da Universidade de São Paulo Brasil

${ }^{3}$ Arquiteto Doutor e Sócio da Vargas Valentim Projetos e Construções Ltda
} 


\title{
A METHOD OF COLLECTION OF TIMES AND TRAVEL ROUTES IN STAIRS AIMING AT OBTAINING WALKING SPEEDS OF CHILDREN IN FIRE DRILLS
}

\begin{abstract}
This article aims at presenting the methods adopted for collection of times at predetermined points and the traveled routes of students along the staircase in fire drills. Then travel distances were also obtained. The equations defined in order to calculate different the travel routes on landings are presented. Those equations considered the dimensions of the landings and the students' average bi-deltoid length. This research was carried out in São Paulo, Brazil, in 2017. A CCTV system was installed in order to collect data. On hundred sixty-four students, from age 6 to 10, were the participants of the fire drill. Four hundred ninety-two travel distances were collected in stair landings and six hundred fiftysix, in its flights. The routes in landings and flights were analyzed separately and it was possible to obtain the frequency of travel routes and the velocity in landings and flights, separately.
\end{abstract}

Key-words: fire drills, fire exits, evacuation routes, walking speed, children.

\section{INTRODUÇÂO}

Os estudos consagrados na literatura relativos às velocidades e fluxos de pessoas utilizados para fundamentar modelos de simulação de abandono, tais como Predtechsnkii e Milinskii (1978) e Fruin (1987), somente consideraram dados relativos à população adulta sadia. Conforme já discutido por alguns autores, os dados relativos às crianças (LÁRUSDÓTTIR, 2013; CUESTA; GWYNNE, 2016; NAJMANOVÁ; RONCHI, 2017; HAMILTON; LENNON; O'RAW, 2017), e outros grupos que podem requerer assistência, como as pessoas com deficiência, (ANDRÉE et al., 2015), e idosos 
(SEKIZAWA, 2004), são ainda pouco explorados e considerados quando se elaboram normas e regulamentações. Sendo assim, diferentes níveis de segurança podem estar sendo oferecidos para uma grande faixa da população, quando o parâmetro utilizado é restrito ao adulto sadio.

Templer (1994), Fujiyama e Tyler (2004), declaram que na área de fisiologia, vários estudos sobre velocidade em superfícies planas foram realizados em ambientes de laboratório. Entretanto, raramente pesquisas sobre velocidade de caminhamento em escadas foram realizadas nas mesmas condições.

Segundo Hoskins (2011), vários estudos apresentam dados relativos à velocidade de pessoas em escadas. No entanto, quando os autores descrevem como eles obtiveram a velocidade, constata-se que diferentes métodos foram utilizados para o seu cálculo. Em percursos de pequena distância, como é o caso dos patamares de escadas, os métodos para coleta dos tempos e dos trajetos são de extrema importância e podem alterar significativamente a velocidade final. Contudo, a maioria dos trabalhos conhecidos não consideram as diversas possibilidades de trajetos nos patamares (PREDTECHSNKII; MILINSKII, 1978; LÁRUSDÓTTIR, 2013; KULIGOWSKI et al., 2015). Esses autores adotam, tanto nos lances quanto nos patamares, os trajetos como sendo único e centralizado. Por outro lado, Cuesta e Gwynne (2016), ampliaram as possibilidades de trajetos nos patamares para três, porém sem detalhar as velocidades resultantes neste elemento (patamares).

Tendo-se em vista os vários métodos adotados por diversos autores, nem sempre apresentados de forma detalhada, nesse artigo, pretendeu-se definir, de forma clara, os critérios adotados para a coleta dos tempos e o trajeto percorrido pelos alunos na escada para uma situação de simulado de abandono. 


\section{OBJETIVO}

Esta pesquisa tem por objetivo discutir o método que foi utilizado para a coleta dos dados (trajetos e tempos) tanto para os lances quanto para os patamares de escadas, e os dados obtidos de trajeto e velocidade num simulado de abandono real.

\section{DESCRIÇÃO DE EDIFÍCIO}

Localizado na cidade de São Paulo, trata-se de uma escola pública municipal de ensino fundamental ${ }^{4}$ (do $1^{\circ}$ ao $9^{\circ}$ ano). O edifício possui três pavimentos (térreo, primeiro e segundo pavimento) sendo que as salas de aula estão localizadas, predominantemente, no primeiro e no segundo pavimento. Os alunos abordados nessa pesquisa estavam no segundo pavimento, exceto a turma do $1^{\circ}$ ano que estava posicionada no primeiro pavimento. $O$ edifício possui duas escadas abertas e que são utilizadas no dia-a-dia pelos alunos. A seguir são apresentadas na Tabela 1 as principais características, do edifício, que interessam à pesquisa.

Tabela 1: Características do Edifício.

Área total construída / Área aproximada do andar tipo

Quantidade de salas de aula por pavimento Largura "bruta" e "livre"

Altura da edificação

Dimensões dos degraus e inclinação

Tipo de piso da escada

a - Na largura "bruta" não se consideram os corrimãos;

b - Trata-se da largura livre utilizada na escada, ou seja, largura bruta descontando-se os corrimãos;

\footnotetext{
${ }^{4}$ No Brasil, a educação básica é o primeiro nível do ensino escolar e compreende três etapas: a educação infantil (para crianças de zero a cinco anos), o ensino fundamental (para alunos de 6 a 14 anos) e o ensino médio (para alunos de 15 a 17 anos)
} 


\section{MÉTODO}

Nos estudos realizados por Ono et al. (2012), Cuesta e Gwynne (2016) e Valentim (2018), constatou-se que diversos trajetos são utilizados nos patamares. Essa variação de trajetos e, consequentemente de distâncias, pode alterar significativamente as velocidades. Dessa forma, nesse estudo, as possibilidades de trajetos nos lances e nos patamares foram ampliadas com 0 objetivo de se obter distâncias de trajetos mais precisas para o cálculo da velocidade.

Esse estudo adotou como premissa três possibilidades de partida e chegada, tanto nos lances quanto nos patamares. Considerou-se que os alunos iniciaram o movimento no patamar, provenientes do lance, pelo caminho interno (I), meio (M) ou externo (E). O critério adotado para o acesso ao lance subsequente, quando da saída do patamar, foi o mesmo. No patamar, também foram adotadas três possibilidades de percurso, ou seja, interno (I), meio (M) ou externo (E). Adicionalmente, para a determinação dos trajetos internos e externos, considerou-se a distância da parede ao eixo do corrimão, $10 \mathrm{~cm}$ entre o ombro do aluno (músculo deltóide) e o eixo do corrimão, definida por Pauls (1980 apud NELSON; MOWRER [14]) e a metade da medida bideltóide do aluno. Na Figura 1 pode-se observar as possibilidades de trajetos considerados nessa pesquisa.

Figura 1: Trajetos nos patamares e lances e possibilidades de percursos no patamar.

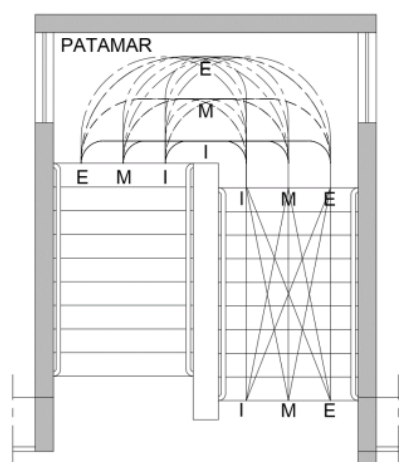

\begin{tabular}{ccccc}
\hline ACESSO & I & M & E & SAÍDA \\
\hline \multirow{3}{*}{ I } & III & IMI & IEI & I \\
& IIM & IMM & IEM & M \\
& IIE & IME & IEE & E \\
\hline \multirow{3}{*}{$\mathbf{M}$} & MII & MMI & MEI & I \\
& MIM & MMM & MEM & M \\
& MIE & MME & MEE & E \\
\hline \multirow{3}{*}{$\mathbf{E}$} & EII & EMI & EEI & I \\
& EIM & EMM & EEM & M \\
& EIE & EME & EEE & E \\
\hline \multicolumn{4}{c}{} & I=interno - M= meio - E=externo
\end{tabular}


Como pode ser observado na Figura 1, embora existam percursos diferentes, algumas distâncias são iguais. Na Figura 2 são apresentadas as variáveis que foram utilizadas nas equações e, na Figura 3, exemplos de equações que foram desenvolvidas para descrever os trajetos percorridos nos lances.

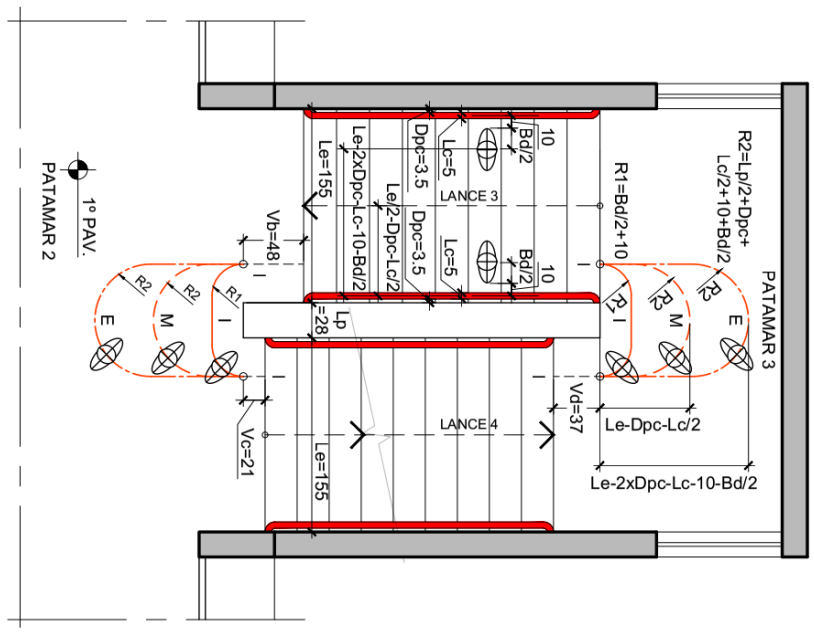

\author{
Legenda: \\ Le: largura total da escada \\ Bd: medida bideltóide dos alunos ${ }^{5}$ \\ Dpc: Distância entre a parede e a face do \\ corrimão \\ Lc: largura do corrimão \\ LP: Largura da parede ou vão entre os lances \\ $\mathrm{Vb}, \mathrm{Vc}, \mathrm{Vd}$ : medidas variáveis \\ I: trajeto pelo lado interno \\ M: trajeto pelo meio \\ $\mathrm{E}$ : trajeto pelo lado externo
}

Figura 2: Variáveis consideradas nas equações que descrevem o percurso no patamar.

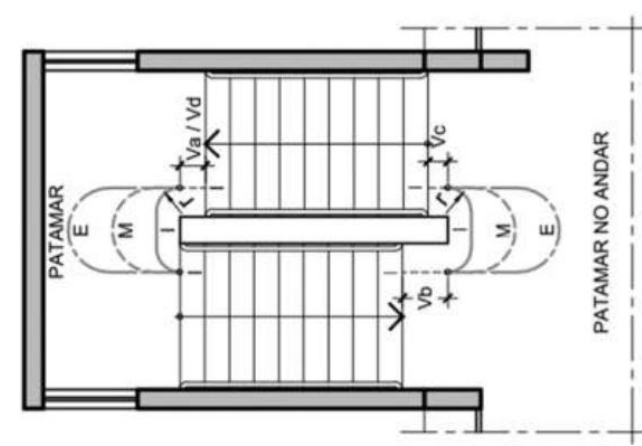

\begin{tabular}{|l|l|}
\hline$I I I=\pi\left(\frac{B d}{2}+10\right)+(L p+2 D p c+L c)+V(1)$ & (1) \\
\hline $\begin{array}{l}I M I=\pi\left(\frac{B d}{2}+10+\frac{L c}{2}+D p c+\frac{L p}{2}\right)+2\left(\frac{L e}{2}-D p c-\frac{L c}{2}\right)-2\left(\frac{B d}{2}+10+\frac{L c}{2}+D p c+\frac{L p}{2}\right) \\
+V(1)\end{array}$ & (2) \\
\hline $\begin{array}{l}I E I=\pi\left(\frac{B d}{2}+10+\frac{L c}{2}+D p c+\frac{L p}{2}\right)+2 L e-2\left(2 D p c+L c+10+\frac{B d}{2}\right) \\
\left.-20+\frac{L c}{2}+D p c+\frac{L p}{2}\right)+V(1)\end{array}$ & (3) \\
\hline $\begin{array}{l}\text { (1) } \mathrm{V} \text { : medida variável } \\
\text { Para percurso no patamar 1: somar } \mathrm{Va} \\
\text { Para percurso no patamar 2: somar } \mathrm{Vb}+\mathrm{Vc}\end{array}$ & \\
\hline
\end{tabular}

Figura 3: Equações que descrevem os percursos III, IMI e IEI.

\footnotetext{
${ }^{5}$ Foi coletada a medida bideltóide de cada aluno. Nas equações, considerou-se a medida bideltóide média de cada uma das classes.
} 


\subsection{Equipamento para coleta}

Para a captura das imagens foram utilizadas câmeras Hikvison, modelo DS-2CE56C0T-IRM, com taxa de 25 fotogramas por segundo. O Digital Video Recorder (DVR) utilizado também foi fabricado pela Hikvison, com capacidade de saída para 16 câmeras. O software para gerenciamento e configuração do sistema de CFTV foi fornecido quando da aquisição do DVR. Na Figura 4 podese observar o posicionamento das câmeras em uma das escadas.
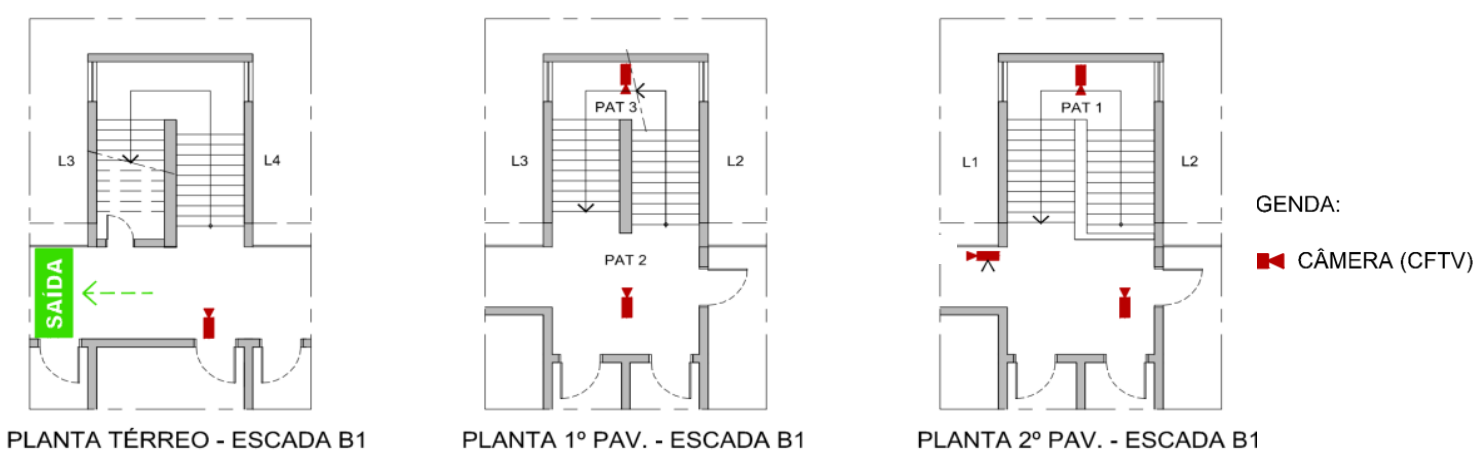

Figura 4: Posicionamento das câmeras de CFTV.

O programa utilizado para leitura dos vídeos foi o Windows Movie Maker. Esse programa permite que a precisão na coleta dos tempos seja mais refinada, pois trabalhamos com precisão de centésimos de segundos, visto que os percursos, tanto nos lances quanto nos patamares, eram curtos.

\subsection{Trajeto percorrido nos lances de escadas}

Para os trechos percorridos nos lances, considerou-se a distância (hipotenusa) entre o primeiro e o último degrau de cada lance. O comprimento de todos os lances foi coletado no local. Na Figura 5 é possível observar em corte a distância considerada para a obtenção da velocidade. Considerou-se como o primeiro tempo coletado no lance o momento em que o pé cruzou a quina do primeiro degrau. Para o último degrau do lance o procedimento foi o mesmo (Ver Figura 5). 

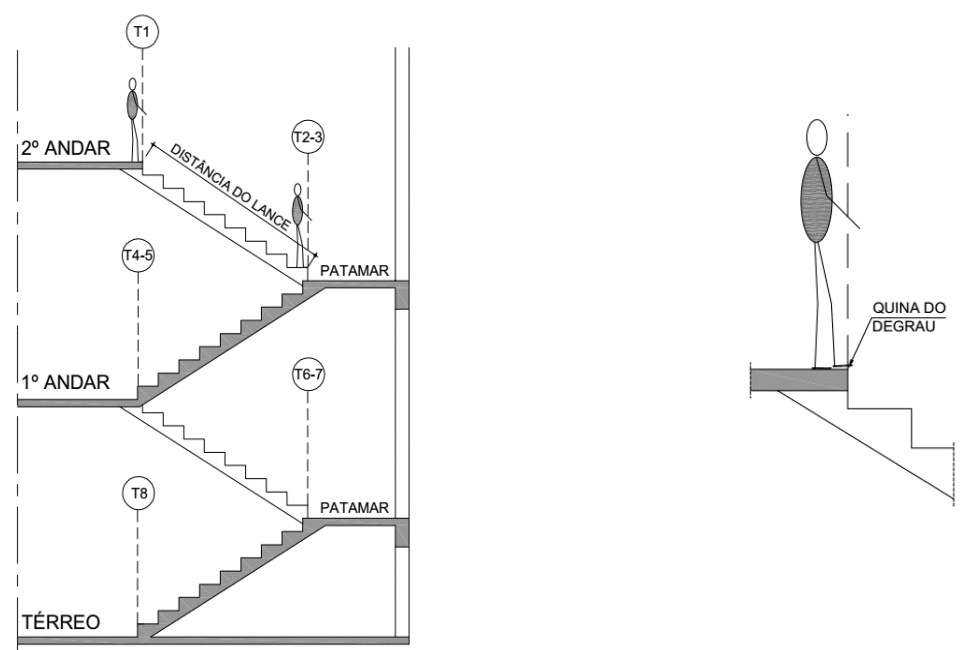

Figura 5: Critério para coleta dos tempos no lance das escadas.

\section{RESULTADOS}

\subsection{Trajetos percorridos e velocidade de caminhamento nos lances}

Conforme já descrito, para cada lance de escada foram coletados dois tempos, no momento da entrada e da saída do lance. A velocidade do aluno no lance foi obtida através da divisão da distância percorrida no lance pelo tempo decorrido entre o início e o fim do trajeto naquele lance. A quantidade de dados de velocidades válidos para os lances foi de 616 .

$\mathrm{Na}$ Figura 6, são apresentados os percursos utilizados pelos alunos durante as descidas nos lances. Tendo-se em vista que em aproximadamente $90 \%$ dos trechos percorridos nos lances, os alunos não mudaram de lado durante a descida (trajeto sempre feito exclusivamente pelo: lado Interno da escada - I; ou lado Externo da escada - E; ou pelo meio da escada - M), considerou-se como a distância de caminhamento, o comprimento de uma linha reta entre o primeiro e o último degrau de cada lance. 


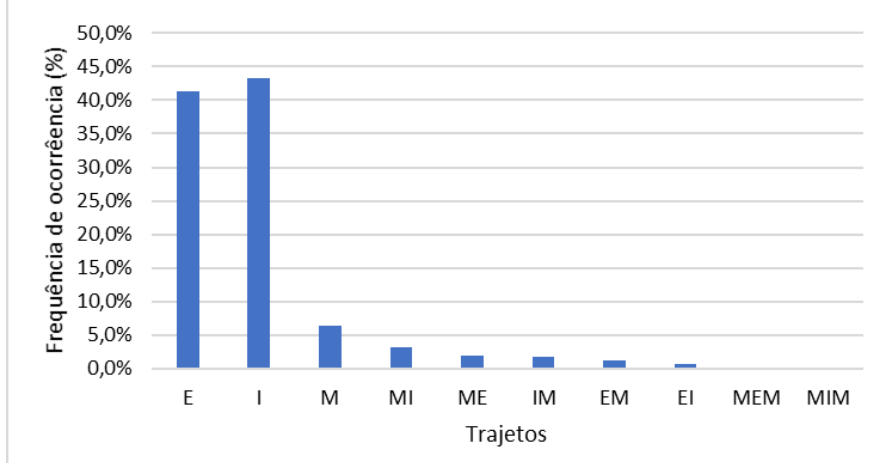

Legenda:
E: externo
I: interno
M: meio
MI: meio e interno
ME: meio e externo
IE: interno e meio
EM: externo e meio
El: externo e interno
MEM: meio, externo e meio
MEM: meio, interno e meio

Figura 6: Percursos utilizados nos lances.

Na Tabela 2, são apresentadas as medidas estatísticas de síntese dos dados contidos nas Figuras 7 e 8: as velocidades médias e o desvio padrão, além do intervalo de confiança de $95 \%$ para a média para os lances, por ano, bem como a quantidade de alunos participantes e lances.

Tabela 2: Dados de velocidade média nos lances por turma

\begin{tabular}{lccccc}
\hline Parâmetro & $1^{\circ}$ ano & $2^{\circ}$ ano & $3^{\circ}$ ano & $4^{\circ}$ ano & $5^{\circ}$ ano \\
\hline Quantidade de alunos & 20 & 35 & 34 & 37 & 37 \\
Lances válidos & $40^{6}$ & 144 & 136 & 148 & 148 \\
Velocidade média (m/s) & 0,72 & 0,89 & 0,95 & 0,93 & 0,82 \\
Desvio padrão (m/s) & 0,14 & 0,28 & 0,21 & 0,36 & 0,29 \\
Intervalo de confiança para a & 0,044 & 0,046 & 0,036 & 0,058 & 0,047 \\
média & & & &
\end{tabular}

Na Figura 7, são apresentados os resumos dos parâmetros estatísticos. A média da amostra é apresentada com a letra " $X$ " e a mediana com uma linha horizontal na área interna da caixa, as bordas superior e inferior da caixa apresentam os limites dos quartis, linhas horizontais nas extremidades inferiores e superiores apresentam os valores extremos e os pontos representam os dados atípicos, ou outliers. Já na Figura 8 são apresentadas as velocidades médias para os lances.

\footnotetext{
${ }^{6}$ Para todas as turmas haviam duas classes, exceto para o $1^{\circ}$ ano que só havia uma. A quantidade de dados para o $1^{\circ}$ ano foi menor, pois eles estavam no primeiro andar do edifício enquanto as demais turmas estavam no segundo andar.
} 


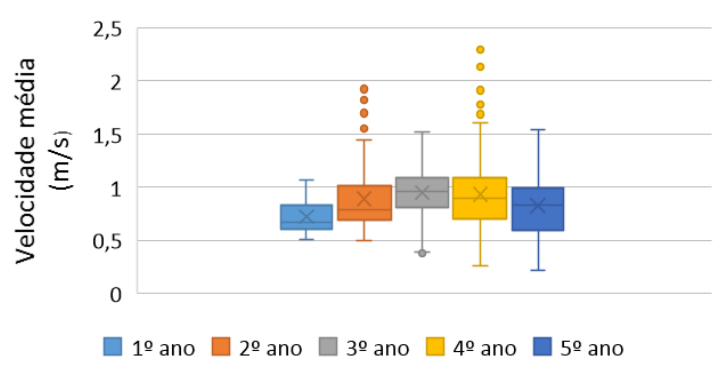

Figura 7: Boxplot demonstrando as velocidades nos lances

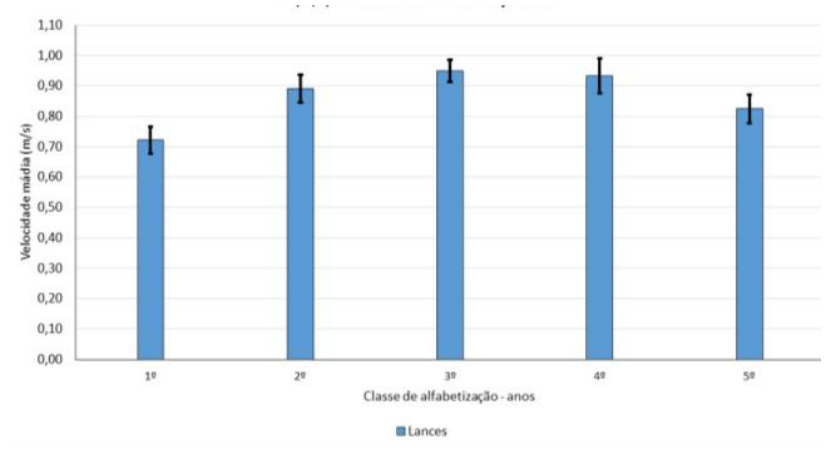

Figura 8: Velocidades médias, com intervalos de confiança de $95 \%$ de probabilidade para os lances.

As menores velocidades foram observadas nos $4^{\circ}$ e $5^{\circ}$ anos, $0,26 \mathrm{~m} / \mathrm{s} \mathrm{e}$ $0,22 \mathrm{~m} / \mathrm{s}$, respectivamente. Essas velocidades são justificadas, visto que a professora do $4^{\circ}$ ano B parou no patamar 3 para organizar a turma e, como consequencia, as duas turmas que desciam a escada na sequência (5ำ ano $\mathrm{A} e$ $5^{\circ}$ ano B), foram obrigadas a diminuir o rítmo e, até mesmo, parar, em função do comportamento desse professor.

Já para os valores máximos, a maior parte dos resultados está entre $1,45 \mathrm{~m} / \mathrm{s}$ e $1,60 \mathrm{~m} / \mathrm{s}$, mas com ocorrência de alguns valores fora desse intervalo, como verificado no $2^{\circ}$ ano $(1,93 \mathrm{~m} / \mathrm{s})$ e no $4^{\circ}$ ano $(2,30 \mathrm{~m} / \mathrm{s})$. Em uma das turmas do $2^{\circ}$ ano, a professora desceu a escada "puxando a fila", no entanto, não se deu conta que alguns alunos pararam no primeiro patamar. Como consequencia, esses alunos que pararam nesse patamar correram para alcançar a professora. Já para os alunos do $4^{\circ}$ ano, em uma das turmas quatro alunos desceram a escada na frente do professor e como eram da primeira turma a descer a escada, estavam com todo o trajeto livre. Mesmo avisados que deveriam caminhar normalmente, esses alunos correram.

A partir dessa dispersão de resultados, buscou-se verificar a hipótese de que a velocidade dos alunos aumentaria com a idade. A Figura 8 apresenta as velocidades médias nos patamares por turmas com os intervalos de confiança de $95 \%$ para a média. Pode-se observar que as velocidades médias com 
intervalo de confiança não apresentaram grande variabilidade entre as turmas, exceto para os alunos do $1^{\circ}$ ano que apresentaram velocidade menor. Por exemplo, os alunos do $2^{\circ}$ e $5^{\circ}$ anos apresentaram velocidades médias semelhantes, ou seja, dentro do intervalo de confiança, assim como os alunos $\operatorname{dos} 2^{\circ}, 3^{\circ}$ e $4^{\circ}$ anos.

\subsection{Trajetos percorridos e velocidade de caminhamento nos patamares}

Com a combinação de três possibilidades de acesso ao patamar, três de percursos no patamar e três de saídas do patamar, obteve-se vinte e sete possibilidades de trajetos. Os trajetos utilizados pelos alunos são apresentados na Figura 9.

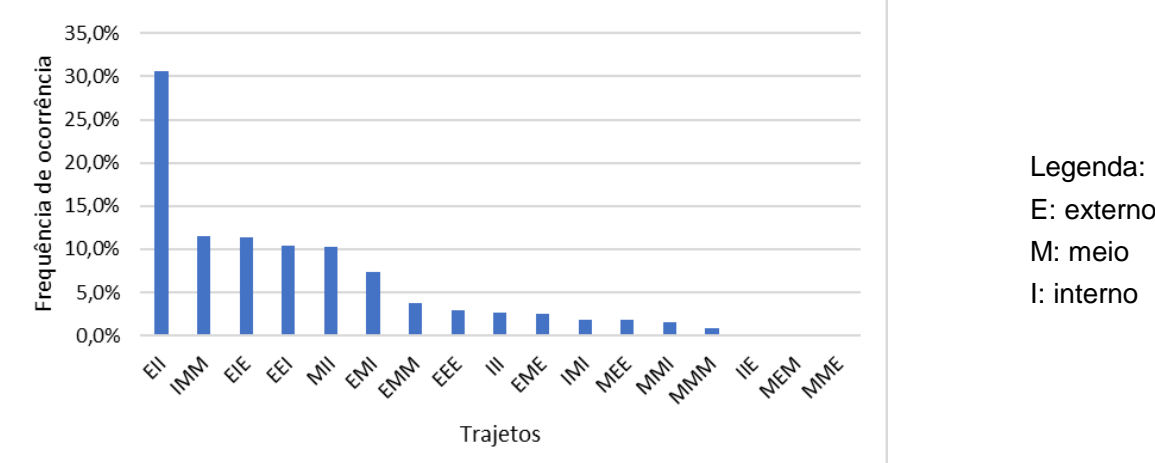

Figura 9: Percursos utilizados nos patamares

A partir dos dados expostos, é possível perceber que as conclusões de Templer (1992 apud KULIGOWSKI et al. 2015) e Predtechenskii e Milinskii (1978), acerca do trajeto percorrido no patamar não se confirmaram nessa pesquisa. Segundo esses autores, os indivíduos tendem a optar por caminhar pelo meio do patamar - o que não ocorreu neste estudo.

$\mathrm{Na}$ Tabela 3, são apresentadas as medidas estatísticas de síntese dos dados contidos nas Figuras 10 e 11. Verificam-se as velocidades médias e o desvio padrão para os patamares, além do intervalo de confiança de $95 \%$ para a média, por ano, bem como a quantidade de alunos participantes e trajetos. 
Tabela 3: Dados de velocidade média nos patamares por turma

\begin{tabular}{llllll}
\hline Parâmetro & 1ํ ano $^{\circ}$ & $2^{\circ}$ ano & $3^{\circ}$ ano & $4^{\circ}$ ano & $5^{\circ}$ ano \\
\hline Quantidade de alunos & 20 & 35 & 34 & 37 & 37 \\
Trajetos nos patamares & 20 & 108 & 102 & 111 & 111 \\
Velocidade média $(\mathrm{m} / \mathrm{s})$ & 0,82 & 0,95 & 0,91 & 0,83 & 0,48 \\
Desvio padrão $(\mathrm{m} / \mathrm{s})$ & 0,34 & 0,50 & 0,43 & 0,39 & 0,22 \\
Intervalo de confiança para & 0,159 & 0,094 & 0,084 & 0,074 & 0,042 \\
a média & & & & &
\end{tabular}

Na Figura 10, são apresentados os resumos dos parâmetros estatísticos e na Figura 11 as velocidades médias para os patamares.

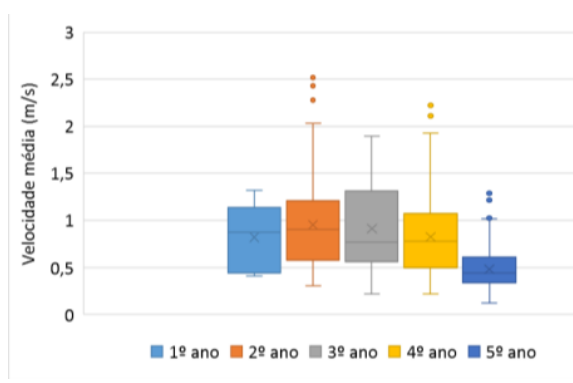

Figura 10: Boxplot demonstrando as velocidades nos patamares

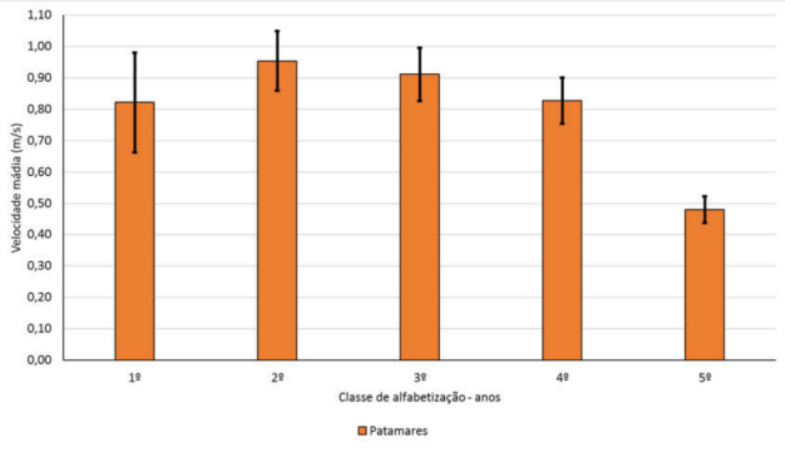

Figura 11: Velocidades médias, com intervalos de confiança de $95 \%$ de probabilidade para os lances

Assim como nos lances, as menores velocidades foram observadas nos $4^{\circ}$ e $5^{\circ}$ anos, $0,22 \mathrm{~m} / \mathrm{s}$ e $0,12 \mathrm{~m} / \mathrm{s}$, respectivamente. Já para os valores máximos, a maior parte dos resultados está entre 1,90 m/s e 2,00 m/s, mas com ocorrência de alguns valores fora desse intervalo, como verificado no $2^{\circ}$ ano $(2,50 \mathrm{~m} / \mathrm{s})$ e no $4^{\circ}$ ano $(2,20 \mathrm{~m} / \mathrm{s})$. Essas velocidades são justificadas pelos mesmos motivos já apresentados para os lances. Assim como nos lances, as velocidades médias com intervalo de confiança não apresentaram grande variabilidade entre as turmas, ou seja, apresentaram velocidades médias semelhantes, dentro do intervalo de confiança.

\section{CONCLUSÕES}

Tinha-se como expectativa encontrar padrões diferenciados de velocidade média para crianças, por idade. Contudo, devido à elevada 
dispersão dos valores obtidos, tanto nos lances quanto nos patamares, não foi possível estabelecer uma relação entre a velocidade média e a idade dos alunos. Vale ressaltar que em simulados de abandono em edifícios escolares e com a participação de crianças, a velocidade por vezes pode ser controlada pelos professores, não proporcionando uma descida constante e uniforme.

Quanto ao trajeto percorrido pelos alunos nos lances das escadas, constatou-se que em, aproximadamente, $90 \%$ dos trechos percorridos, os alunos não mudaram de lado durante a descida, ou seja, seguiram uma trajetória retilínea entre o primeiro e o último degrau de cada lance.

Com relação aos trajetos percorridos nos patamares, para todas as turmas que participaram do experimento, menos de $1 \%$ dos alunos adotaram 0 trajeto MMM. Portanto, a adoção de um único trajeto nos patamares, (metodologia adotada por diversos pesquisadores citados neste estudo) pode levar a subestimar ou superestimar as distâncias percorridas e, consequentemente, resultar em velocidades de evacuação muito diferentes do real. Essas informações (a frequência dos caminhos percorridos) podem ajudar os desenvolvedores a construir softwares de simulação de evacuação que reproduzam melhor a realidade do comportamento de crianças.

AGRADECIMENTOS: Os autores agradecem à FAPESP (Processo 2015/26153-3) pelo apoio à pesquisa.

\section{REFERÊNCIAS}

PREDTECHENSKII; V. M.; MILINSKII, A. I. Planning for Foot Traffic in Building. New Delhi: Amerind Publishing, Co. Pvt. Ltd, 1978.

FRUIN, J. J. Pedestrian Planning and Design (Revised Edition). Mobile: Elevator World, 1987.

LÁRUSDÓTTIR, A. R. Evacuation of Children: Focusing on daycare centers and elementary schools. Technical University of Denmark, Department of Civil Engineering. (BYG Rapport R-295), 2013.

CUESTA, A; GWYNNE, S. M. V. The collection and compilation of school evacuation data for model use. Safety Science. V. 84, p. 24-36, 2016. 
NAJMANOVÁ, H, RONCHI E. An experimental data-set on pre-school children evacuation. Fire Technology, 2017, 53, pp. 1509-1533.

HAMILTON, G. N.; LENNON, P. F.; O'RAW, J. Human behaviour during evacuation of primary schools: Investigations on pre-evacuation times, movement on stairways and movement on the horizontal plane. Fire Safety Journal, v. 91, p. 937-946, 2017.

ANDRÉE et al. Attitudes about safe refuge areas as an egress strategy from the point of view of the mobility impaired people, authorities and building planners. In: Grd International Symposium on Human Behaviour in Fire, 2015, Cambridge, UK.

SEKIZAWA, A. Care of vulnerable populations: Who are vulnerable to fires and what care is needed for their fire safety? In: 3rd International Symposium on Human Behaviour in Fire, 2004, London, UK.

TEMPLER, J. (1995). The Staircase: Studies of hazards, falls, and safer design. MIT Press, 1995.

FUJIYAMA, T.; TYLER, N. Pedestrian speeds on stairs -Initial step for simulation model. In: Proceedings of 36 th Universities Transport Studies Group Conference, Life Science Centre, 2004, Newcastle upon Tyne, UK.

HOSKINS, B. L. The effects of interactions and individual characteristics on egress down stairs. Thesis, (Doctor of Science in Fire Protection Engineering) - University of Maryland, College Park, Maryland, 2011.

KULIGOWSKI, E. D.; et al. Movement on Stairs During Building Evacuations. National Institute of Standards and Technology, 2015. Disponível em <https://ws680.nist.gov/publication/get_pdf.cfm?pub_id=902502>. Acesso em 011 de Jul. 2017.

ONO, R., VALENTIN M.V., VITTORINO. F. Walking speed data off fire drills at an elementary school. In: 5rd International Symposium on Human Behaviour in Fire, 2012, Cambridge, UK.

NELSON, H. E.; MOWRER, F. W. Emergency Movement. In: SFPE Handbook of Fire Protection Engineering, Third Edition, Society of Fire Protection Engineers, Bethesda, MD, 2002. Section 3, Chapter 14.

VALENTIM, M. V. Comportamento de crianças em movimento em escadas: Subsídios para o dimensionamento dos meios de escape em escolas. Tese (Doutorado), São Paulo, Faculdade de Arquitetura de Urbanismo da Universidade de São Paulo, 2018. 